\title{
Primary Large Cell Neuroendocrine Carcinoma of Buccal Mucosa: A Report of a Case at an Unusual Location
}

\author{
Girish Anandrao Kadkol, Madhura Devdatta Phadke, Zeba Nisar*and Jaydeep Nilkanthrao Pol \\ The Oncopathology Centre, Mahatma Gandhi Cancer Hospital, Miraj.
}

\begin{abstract}
Neuroendocrine carcinoma is a poorly differentiated carcinoma that usually occurs in the lung. Large cell neuroendocrine carcinoma is a Non-small cell lung carcinoma that shows histological features of neuroendocrine morphology (including rosettes and peripheral palisading) and expresses immunohistochemical neuroendocrine markers.

Oral cavity is an exceedingly rare site for primary Neuroendocrine Carcinoma. This case report describes Primary Large Cell Neuroendocrine Carcinoma (LCNEC) of buccal mucosa and lower gingiva in a 65 years old male.

To the best of the author's knowledge this is the third case of intraoral LCNEC \& second case with LCNEC in buccal mucosa to date in English literature. Thus far, only a small number of cases of intraoral mucosal Neuroendocrine carcinoma have been reported and no definitive standard treatment strategy has been determined.
\end{abstract}

\section{Keywords: Intraoral, Large Cell Neuroendocrine Carcinoma, Buccal Mucosa}

\section{Introduction}

Tumors of the neuroendocrine system constitute a heterogeneous group of lesions that vary in origin, location, histological appearance, the degree of differentiation, biological behavior, functional activity and size but share certain histochemical, immunohistochemical, and ultrastructural characteristics. ${ }^{[1]}$ Large cell neuroendocrine carcinoma (LCNEC) is a high-grade neuroendocrine tumor that was first detected in the lung as part of the spectrum of pulmonary neuroendocrine tumors. Although originally found in the lung, LCNEC has since been described in a variety of extrapulmonary locations. There are reports of these tumors arising in head and neck region, the commonest being larynx..$^{[2]}$ Salivary glands are perhaps the second most common site, whereas intraoral mucosa is one of the rarest sites for a primary NECs ${ }^{[3]}$ Primary intraoral mucosal LCNEC is a rare neoplasm with only 2 cases reported in the English literature to date. ${ }^{[4,5]}$ This paucity of data means that a definitive therapeutic strategy has yet to be determined. The present study reports a rare case of LCNEC of the buccal mucosa and gingiva and reviews the clinicopathological characteristics of this uncommon tumor type.

\section{Case Report}

65 years old male patient with history of smoking, Diabetes Mellitus \& Hypertension presented with non healing spongy ulcerative lesion over right buccal mucosa and lower alveolus since 4 months. Patient had very poor general condition. Also, he had enlarged right cervical lymph node which on FNAC showed positivity for malignant cells suggestive of metastatic carcinoma. Incisional biopsy was done, specimen consisted of irregular grey-white soft tissue pieces which on microscopy showed bits of tissue lined by stratified squamous epithelium. The submucosal tissue showed a tumour composed of large round to oval cells with enlarged hyperchromatic nuclei with dispersed chromatin, some having prominent nucleoli and having scanty cytoplasm arranged in sheets and clusters. Increased mitosis and crushing was seen. The intervening stroma showed diffuse leucocytic infiltration. Histomorphological features were consistent with Poorly differentiated carcinoma(fig.1:a,b\&c).

On Immunohistochemistry, the tumour cells stained positive for CK, NSE, Synaptophysin, and Chromogranin (Fig.2:a,b\&C). Mib1 index was high(80\%). CK20 was negative. Hence, a diagnosis of LCNEC was confirmed.

\section{Discussion}

The WHO classification of laryngeal NECs is derived from that for lung NECs. This has been extrapolated to other head and neck site primary mucosal NECs and comprises tumour types: Well-differentiated neuroendocrine carcinoma, Moderately differentiated neuroendocrine carcinoma and Poorly differentiated neuroendocrine carcinoma. Poorly differentiated NEC is further subclassified into Small 
Table 1: Reported cases of intra oral mucosal LCNEC.

\begin{tabular}{|l|l|l|}
\hline Year & Reference & Site \\
\hline 2009 & Kusafuka K et al. ${ }^{[4]}$ & Tongue base \\
\hline 2011 & Krishnamurthy A. et al ${ }^{[5]}$ & Retromolar Trigone \\
\hline 2017 & Present case & Right buccal mucosa and lower alveolus \\
\hline
\end{tabular}

Table 2: Immunohistochemical profile of neuroendocrine tumours.

\begin{tabular}{|l|c|c|c|c|c|}
\hline \multirow{2}{*}{ Antibody } & Result & \multicolumn{3}{|c|}{ Expected percentage (\%) positivity in pulmonary NE tumors } \\
\cline { 2 - 6 } & Present case & LCNEC & SmCNEC & Typical carcinoid & Atypical carcinoid \\
\hline Keratin & 90 & $>85$ & 100 & 56 & $30-40$ \\
\hline NSE & 90 & 95 & 95 & 95 & 95 \\
\hline Chromogranin & 70 & 80 & 50 & 100 & 100 \\
\hline Synaptophysin & 80 & 40 & 100 & 84 & 80 \\
\hline
\end{tabular}

NSE: Neuron Specific Enolase

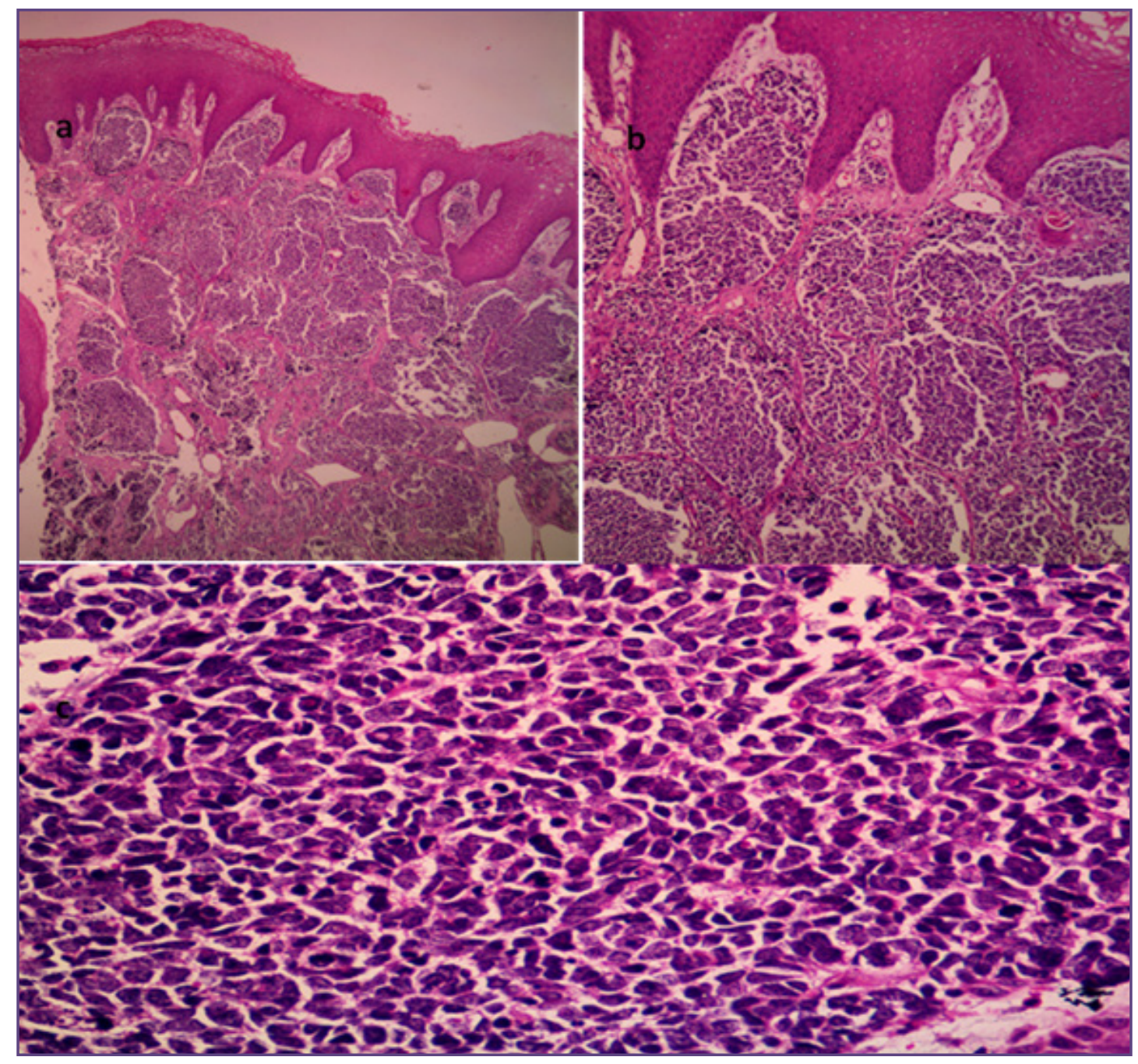

Fig.1: a (4X, H\&E stained): Tissue bit lined by stratified squamous epithelium and tumour in submucosa. b (10X, H\&E stained): Tumour cells arranged in sheets and clusters in submucosa. c (40X, H\&E stained): Large round to oval cells with enlarged hyperchromatic nuclei with dispersed chromatin, some having prominent nucleoli and scanty cytoplasm. 


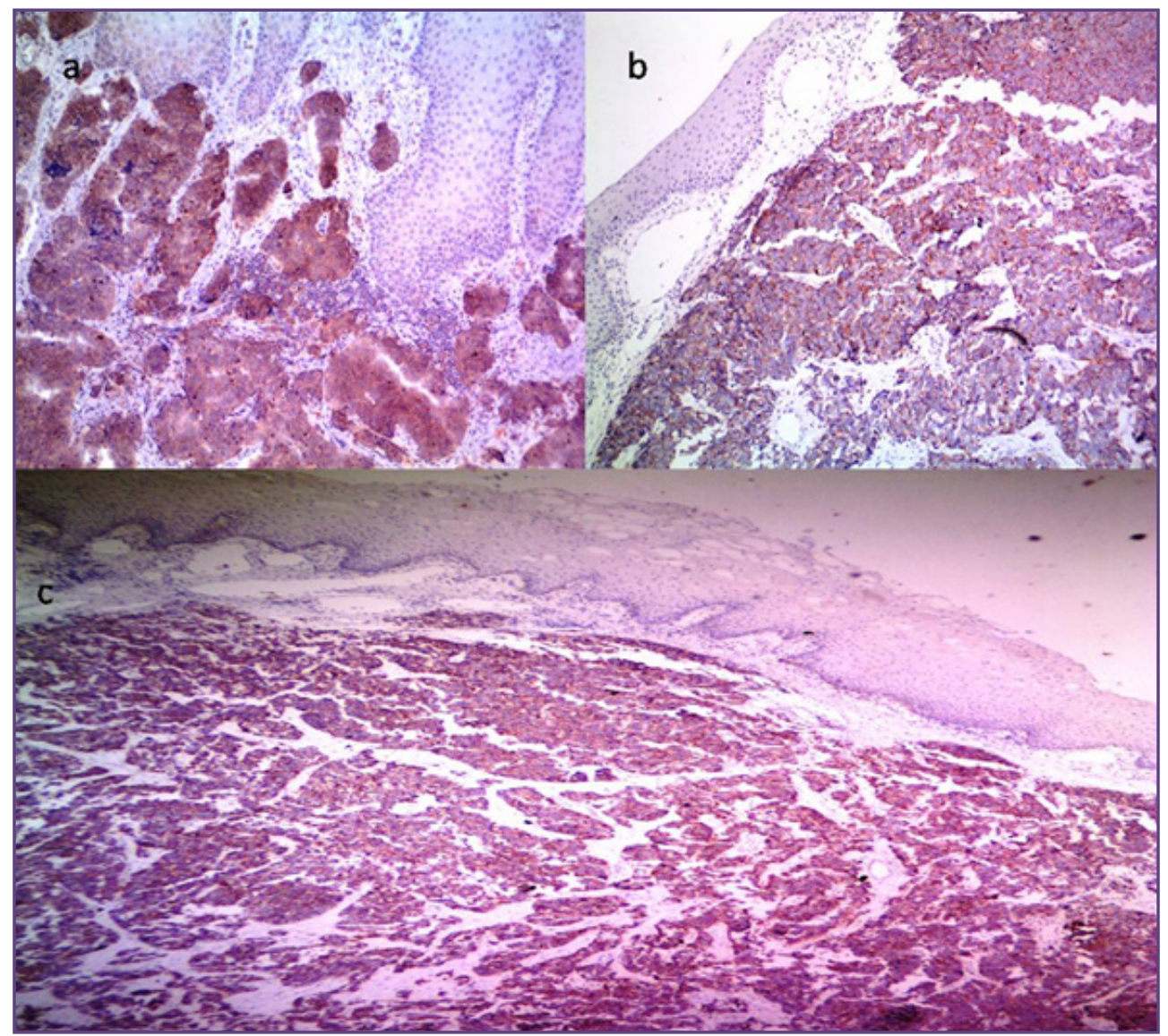

Fig. 2: Tumour cells expressed- a- NSE, b- Synaptophysin, c- Chromogranin (10X).

cell neuroendocrine carcinoma (SmCNEC) and large cell neuroendocrine carcinoma. ${ }^{[6]}$

Only 18 NETs originating in the oral mucosa have been reported in the English literature ; of these, SmCNEC were the most common ( 9 cases), followed by Merkel cell carcinoma (3 cases), LCNEC (2 cases),Moderately differentiated NEC (1 case), Typical carcinoid (1 case) and other (2). Of these intraoral mucosal NETs, most common site was Tongue (9 cases) followed by Cheek/ buccal mucosa (4 cases), Gingiva (3 cases), Retromolar Trigone ( 2 cases), floor of mouth (1 case) and Mucobuccal fold( 1 case). In the two reported cases of LCNEC of oral mucosa ${ }^{[4,5]}$ the primary sites were the tongue base and retromolar trigone(Table1).

SmCNEC of the oropharynx affects mostly men, mean age 61 years with a heavy smoking history. ${ }^{[7]}$ It is a characteristic feature that these tumors at their primary site are small and are devoid of clinical symptoms till the tumor has metastasized. Interestingly, our patient was a male, smoker, aged 65 years and presented with a small ulceroproliferative lesion at the right buccal mucosa and lower gingival with enlarged neck node. He had metastasis to ipsilateral lymph nodes at the time of diagnosis, but no evidence of metastatic disease elsewhere. Other institutions have reported cervical adenopathy in $80 \%$ of patients of primary NEC of head and neck at the time of diagnosis. ${ }^{[8]}$ Neuroendocrine tumors were formally diagnosed as poorly differentiated SCCs and previous reports have pointed out that neuroendocrine tumor was often misdiagnosed as poorly differentiated SCC or poorly differentiated adenocarcinoma. ${ }^{[9]} \mathrm{IHC}$ hence become essential for the demonstration of neuroendocrine nature of these tumours (Table2). The diagnosis of LCNEC of present case was based on IHC features.

Merkel cell carcinoma is rare and more frequent in skin, though it has also been described intra-orally. In present case, CK 20 is negative thus excluding the possibility of Merkel cell carcinoma. ${ }^{[1]}$ The possibility that the current tumor was a metastatic LCNEC was dismissed, as extensive clinical investigation failed to detect a primary tumor site.

To the best of our knowledge, within the category of LCNECs as primary oral cavity tumors, there exist only 3 
cases of LCNEC including the present case in the English literature.[4,5] Currently, well-established treatment protocols do not exist. Surgery alone is inadequate because these tumors tend to progress rapidly.

\section{Conclusion}

As we have discussed LCNEC of oral mucosa is extremely rare clinicians should be aware of this possibility, particularly in elderly men with a history of heavy smoking. In addition to histopathological examination and IHC, clinical evaluation of the patient is necessary to exclude a diagnosis of a metastatic tumor. Because of rarity of this entity studies are difficult to be conducted to define standard treatment. The aggressive nature of this tumour type at other sites is well known. Thus, it requires an early diagnosis and mandates surgical resection. It is important to report single institution case of LCNEC of the oral cavity, to perhaps aid in forming a consensus on the treatment.

\section{Reference}

1. Coleman H., Kennedy M., Altini M., Crooks J., Tsakiris P. Neuroendocrine (carcinoid) tumor of the mandible: a case report and review of the literature. Oral Surgery, Oral Medicine, Oral Pathology, Oral Radiology, and Endodontics. 1996;81(1):74-78.

2. Mills, S.E. Neuroectodermal Neoplasms of the Head and Neck with Emphasis on Neuroendocrine Carcinomas. Modern Pathology, 2002:15, 264-278.

3. Hull MT, Eble JN, Warfel KA. Extrapulmonary oat-cell carcinoma of the tongue: An electron-microscopic study. J Oral Pathol. 1984;13:489-96.
4. Kusafuka K, Asano R, Kamijo $\mathrm{T}$, et al. Large cell neuroendocrine carcinoma of the tongue base: Case report of an unusual location with immunohistochemical analysis. Int J Oral Maxillofac surg, 2009 Mar;38(3):296-9.

5. Krishnamurthy A, Vaidhyanathan A, Majhi U. Large cell neuroendocrine carcinoma of the retromolar trigone. J Can Res Ther 2011;7:343-6.

6. Adel K. El-Naggar, John K.C. Chan, Jennifer R. Grandis, Takashi Takata, Pieter J. Slootweg. WHO Classfication of Head and Neck Tumours. 4th Edition. International Agency for Research on Cancer (IARC), Lyon, France. 2017

7. Bishop JA, Westra WH. Human papillomavirus-related small cell carcinoma of the oropharynx. Am J Surg Pathol 2011 nov;35:1679-84.

8. Hatoum GF, Patton B, Takita C, et al. Small cell carcinoma of the head and neck: the University of Miami experience. Int J Radiat Oncol Biol Phys 2009 june 1;74(2):477-81.

9. Jiang SX, Kameya T, Shoji $M$, et al. Large cell neuroendocrine carcinoma of the lung: A histologic and immunohistochemical study of 22 cases. Am J Surg Pathol.1998 May; 22(5):526-37.

10. Travis WD, Linnoila RI, Tsokos MG,et al. Neuroendocrine tumors of the lung with proposed criteria for large-cell neuroendocrine carcinoma. An ultrastructural, immunohistochemical, and flow cytometric study of 35 cases. Am J Surg Pathol.1991 jun(6);15:529-53.

11. Barrett AW, Cort EM, Patel P, Berkovitz BK. 2000. An immunohistological study of cytokeratin 20 in human and mammalian oral epithelium. Arch Oral Biol 45:879-887.

*Corresponding author:

Dr. Zeba Nisar, The Oncopathology Centre, Mahatma Gandhi Cancer Hospital, Near Gulabrao Patil Homeopathy College, Miraj District- Sangli (M.S.) Pin-416410, India

Phone: +919644635542

Email: dr.zeba.ahmed@gmail.com

Financial or other Competing Interests: None. 